\title{
Influence de la saturation des niveaux d'impuretés sur la dynamique de la luminescence de couches épitaxiées de nitrure de gallium
}

\author{
H. Haag, B. Hönerlage, P. Gilliot et R. Lévy \\ Groupe d'Optique Non Linéaire et d'Optoélectronique, Institut de Physique et Chimie \\ des Matériaux de Strasbourg, UMR 7504 du CNRS, ULP, 23 rue du Loess, BP. 20 CR, \\ 67037 Strasbourg cedex, France
}

\begin{abstract}
Résumé : Nous avons étudié la dynamique de la luminescence de couches épitaxiées de nitrure de gallium sur substrat de saphir. Sous fortes intensités d'excitation, nous observons une augmentation du temps de recombinaison de la luminescence jaune. Ces résultats s'interprètent à l'aide de niveaux d'impuretés situés $1 \mathrm{eV}$ au-dessus de la bande de valence, jouant un rôle de réservoir saturable.
\end{abstract}

\section{INTRODUCTION}

Le nitrure de gallium est un semi-conducteur dont le gap est de $3,5 \mathrm{eV}$ à température ambiante [1]. Il suscite actuellement un vif intérêt pour diverses raisons. Tout d'abord, ce matériau permet la fabrication de diodes et de lasers émettant dans le bleu [2]. L'utilisation de lasers bleus présente plusieurs avantages, dont le plus important concerne le stockage de l'information. En effet, plus la longueur d'onde du faisceau est faible et plus sa tache de focalisation sera petite. Avec une diode laser bleue émettant à $350 \mathrm{~nm}$, il serait ainsi possible de stocker quatre fois plus d'informations, à surface égale, qu'avec les diodes laser rouges actuelles émettant à $635 \mathrm{~nm}$. D' autres applications sont également possibles [3], notamment dans le domaine de l'éclairage domestique puisque, à l'aide de diodes électroluminescentes à base de nitrure, il est désormais possible de recréer l'intégralité du spectre visible. En effet, les alliages GaInN et GaAlN permettent de couvrir la région de 1,95 à $6,20 \mathrm{eV}$ de manière continue suivant la concentration d'indium ou d'aluminium. L'enjeu commercial est considérable lorsque l'on sait que les diodes électroluminescentes consomment deux fois moins d'énerğie que les ampoules à incandescence classiques et que leur durée de vie pourrait être de plusieurs années. Actuellement, la durée de vie des lasers bleus est relativement limitée [4] (environ 1000 h), ce qui est probablement dû à la mauvaise qualité cristalline des couches.

Historiquement, le développement des lasers à base de GaAs et de $\mathrm{ZnSe}$ a montré que la compréhension de la dynamique de recombinaison des porteurs a été le facteur clé pour la réalisation de lasers à semi-conducteurs. Nous pouvons supposer qu'il en sera de même pour les nitrures de la famille III-V. C'est dans ce cadre que se situent nos travaux.

Notre étude porte sur un échantillon de GaN de $2 \mu \mathrm{m}$ d'épaisseur, obtenu par MOCVD sur substrat de saphir. Il existe un important désaccord de maille entre la couche de GaN et 
son substrat (13\%), ce qui induit un nombre important de défauts structuraux. Ils donnent lieu à des états pièges situés environ $2,3 \mathrm{eV}$ au-dessus de la bande de valence. Ces niveaux sont responsables d'une large bande d'émission dans le jaune. La figure 1 présente le spectre de luminescence d'un échantillon de GaN où nous pouvons observer, outre la luminescence jaune, une forte raie d'émission dans le proche UV $(3,42 \mathrm{eV})$ correspondant à la recombinaison radiative des porteurs de la bande de conduction avec les trous de la bande de valence. Notre but est de déterminer la dynamique de la luminescence jaune et de la luminescence bleue et de les comparer.

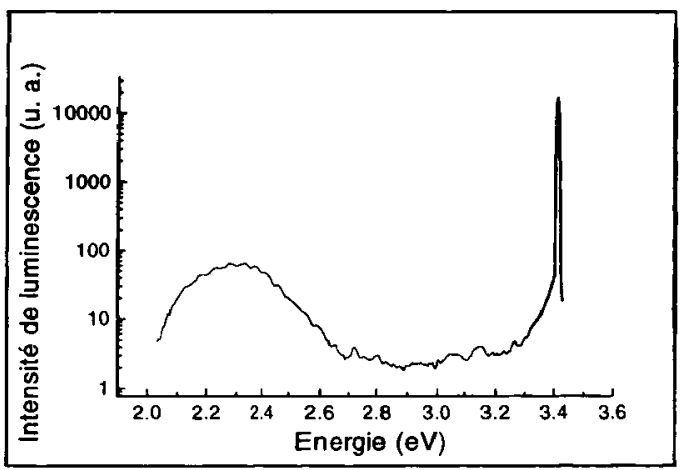

Figure 1 : Luminescence intégrée en temps d'un échantillon de GaN.

\section{RÉSULTATS ET DISCUSSION}

Nous excitons l'échantillon à une énergie de photon de 4,66 eV avec la quatrième harmonique d'un laser $\mathrm{Nd}$ : YAG pulsé, émettant des impulsions de $25 \mathrm{ps}$ à $5 \mathrm{~Hz}$. Le signal de luminescence est mesuré par une caméra à balayage de fente (Streak camera) avec une résolution temporelle de 5 ps. Nous séparons la luminescence bleue de la luminescence jaune à l'aide de filtres interférentiels et étudions, à température ambiante, la dynamique des luminescences bleues et jaunes pour différentes intensités d'excitation.

Nous observons deux régimes d'excitation. Pour des faibles intensités $\left(\mathrm{I}<500 \mathrm{~kW} . \mathrm{cm}^{-2}\right)$ nous ajustons le comportement temporel de la luminescence bleue par une bi-exponentielle, dont les temps caractéristiques sont $\tau_{1}=25 \mathrm{ps}$ et $\tau_{2}=60 \mathrm{ps}$. Le temps de déclin de la luminescence jaune est inférieur à notre résolution expérimentale, limitée par la durée des impulsions laser. Pour de plus fortes intensités d'excitation $\left(\mathrm{I}>500 \mathrm{~kW} \mathrm{~cm}^{-2}\right)$, nous observons un comportement de seuil. Il apparaît en effet une brusque augmentation de $\tau_{2}$ qui atteint $170 \mathrm{ps}$ pour une intensité de $1 \mathrm{MW} \mathrm{cm}^{-2}$ (cf. figure 2). Pour ces mêmes intensités, la luminescence jaune présente un temps de déclin de $430 \mathrm{ps}$ (cf. figure 3 ). Ce comportement est manifestement dû à une saturation de niveaux.

Pour rendre compte du comportement observé nous devons considérer, outre les états de bandes et les niveaux des états pièges responsables de la luminescence jaune, des niveaux d'impuretés dont plusieurs travaux ont montré l'existence [5]. Ces niveaux correspondraient à des centres profonds, situés $1 \mathrm{eV}$ au-dessus de la bande de valence et seraient dus à des impuretés (oxygène ou carbone) ou à des défauts structuraux [6] (centres d'inversions). À faible intensité, ils joueraient le rôle d'un canal de recombinaison non radiatif des porteurs 
concurrent du canal responsable de la luminescence jaune. Par conséquent, nous modélisons, notre expérience par le système d'équations couplées suivant (cf. figure 4):

$$
\left\{\begin{array}{l}
\frac{d n_{C}}{d t}=G(t)-\frac{1}{\tau_{C V}} n_{C}-\frac{1}{\tau_{C P}} n_{C}-\frac{1}{\tau_{C l}} n_{C}\left(1-\frac{n_{I}}{n_{I}^{0}}\right) \\
\frac{d n_{P}}{d t}=\frac{1}{\tau_{C P}} n_{C}-\frac{1}{\tau_{P V}} n_{P}-\frac{1}{\tau_{P I}} n_{P}\left(1-\frac{n_{I}}{n_{I}^{0}}\right) \\
\frac{d n_{I}}{d t}=\frac{1}{\tau_{C I}} n_{C}\left(1-\frac{n_{l}}{n_{I}^{0}}\right)+\frac{1}{\tau_{P I}} n_{P}\left(1-\frac{n_{I}}{n_{l}^{0}}\right)-\frac{1}{\tau_{N V}} n_{I}
\end{array}\right.
$$

où $n_{C}, n_{P}$ et $n_{I}$ représentent respectivement la densité de porteurs de la bande de conduction, des états pièges $(2,4 \mathrm{eV})$ et des états d'impuretés $(1,0 \mathrm{eV}) . \tau_{\mathrm{CP}}$ représente le temps de relaxation de la bande de conduction vers les états pièges, $\tau_{\mathrm{Cl}}$ celui de la bande de conduction vers les états d'impuretés, $\tau_{\mathrm{CV}}$ celui de la bande de conduction vers la bande de valence, $\tau_{\mathrm{PV}}$ celui des états pièges vers la bande de valence, $\tau_{\mathrm{PI}}$ celui des états pièges vers les états d'impuretés et $\tau_{\mathrm{IV}}$ celui des états d'impuretés vers la bande de valence. $G(\mathrm{t})$ décrit la génération des porteurs par l'excitation lumineuse et $n_{l}^{0}$ représente la densité d'impuretés.

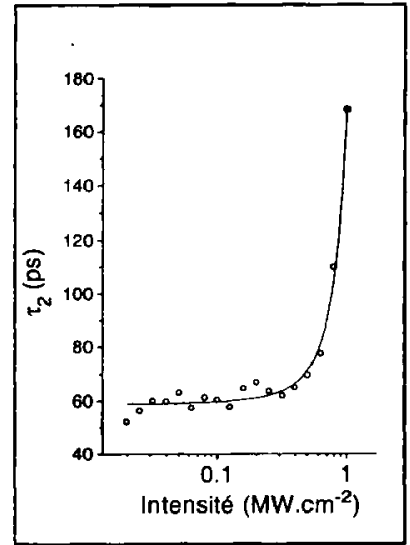

Figure 2 : Second temps de recombinaison de la luminescence bleue en fonction de l'intensité d'excitation.

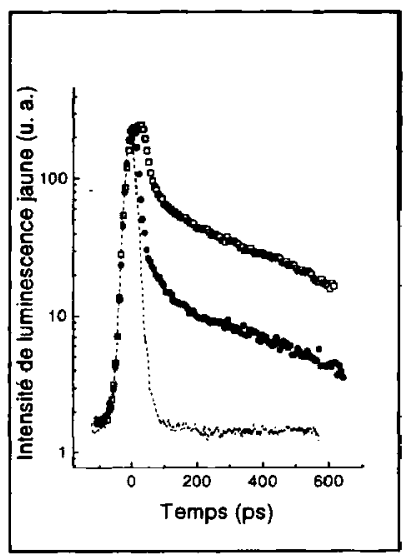

Figure 3 : Variation temporelle de la luminescence jaune pour différentes intensités (carrés: $650 \mathrm{~kW} . \mathrm{cm}^{-2}$, ronds: $550 \mathrm{~kW} . \mathrm{cm}^{-2}$, pointillés: $400 \mathrm{~kW} . \mathrm{cm}^{-2}$ ).

À faible intensité, la solution du système (1) pour la densité de porteurs de la bande de conduction $\mathrm{n}_{\mathrm{C}}$ est une double exponentielle, ce qui est en accord avec l'expérience. Le temps de recombinaison des porteurs de la bande de conduction ayant été déterminé précédemment par des mesures de mélange d'ondes [7], nous attribuons le temps de $25 \mathrm{ps}$ au temps de relaxation des porteurs de la bande de conduction vers les différents états localisés $\left(\tau^{-1}=\tau_{C P}^{-1}+\tau_{C I}^{-1}\right)$. La courte durée de ce processus laisse supposer une forte influence des processus non radiatifs (des temps de l'ordre de dix nanosecondes sont prédits théoriquement pour des échantillons de très bonne qualité). Au vu du temps de recombinaison de la luminescence jaune, nous pouvons supposer qu'il en est de même pour les porteurs piégés, qui se recombinent principalement de manière non radiative. 
Pour de fortes intensités, la saturation des niveaux profonds supprime largement le canal non radiatif $\left(n_{I}=n_{l}^{0}\right)$, ce qui rend accessibles les temps $\tau_{C P}$ et $\tau_{P V}$. Nous obtenons $\tau_{\mathrm{CV}} \geq 170$ ps et $\tau_{\mathrm{PV}}=430$ ps.

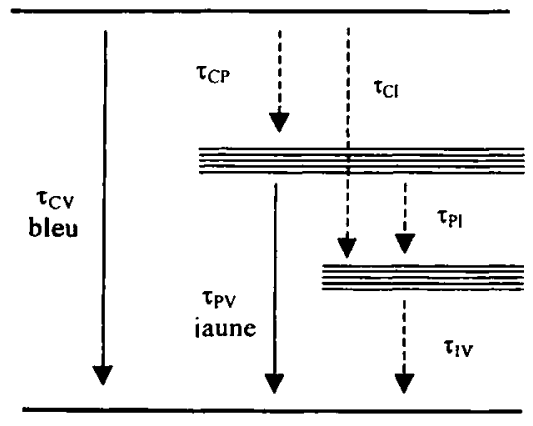

Bande de conduction $(3,42 \mathrm{eV})$

Etats pièges $(2,4 \mathrm{eV})$

Etats d'impuretés $(1,0 \mathrm{eV})$

Bande de valence

Figure 4 : Schéma de niveaux dans le nitrure de gallium. Traits pleins : recombinaison radiative. Tirets : recombinaison non radiative.

\section{CONCLUSION}

Nous avons étudié la dynamique de la luminescence du nitrure de gallium en régime picoseconde. Pour de faibles intensités d'excitation, la luminescence bleue décroît de manière bi-exponentielle avec des temps de relaxation de 25 et de 60 ps. Le temps de déclin de la luminescence jaune est très rapide, ce qui laisse supposer un rôle important des processus non radiatifs. Ce canal serait, entre autres, dû à des centres profonds, situés $1 \mathrm{eV}$ au-dessus de la bande de valence. À forte intensité d'excitation, nous avons mis en évidence la saturation de ces niveaux.

\section{Remerciements.}

Ce travail a été effectué sur des échantillons élaborés au groupe d'étude des semiconducteurs de Montpellier, par Messieurs O. Briot et R.L. Aulombard. Nous tenons à les remercier pour leur collaboration.

\section{Références}

[1] Monemar B., Phys. Rev. B, 10 (1974) 676.

[2] Rigby P., Nature, 384 (1996) 610.

[3] Nakamura S et Fasol G. "The blue laser diode - GaN based light emitters and lasers", Springer-Verlag Berlin Heidelberg (1997).

[4] Nakamura S., Mukai T et Senoh M., Appl.Phys. Lett. 64 (1994) 1687.

[5] Kim D., Libon I. H., Voelkmann C., Shen Y. R. et Petrova-Koch V., Phys. Rev. B 55 (1997) 4907.

[6] Calleja E., Sánchez F.J., Basak D., Sánchez-García M.A., Muñoz E., Izpura I., Calle F., Tijero J. M. G., Beamont B., Lorenzini P. et Gibart P., Phys. Rev. B 55 (1997) 4689.

[7] Haag H., Petit S., Gilliot P., Lévy R., Briot O., et Aulombard R.L., Mat. Sci. and Eng. B 50, 197 (1997). 